\title{
Organometallic nanoprobe to enhance optical response on the polycyclic aromatic hydrocarbon benzo[a]pyrene immunoassay using SERS technology
}

\author{
Dribek Mohamed ${ }^{1,3}$, Rinnert Emmanuel ${ }^{1,{ }^{*}}$, Colas Florent ${ }^{1}$, Crassous Marie-Pierre ${ }^{1}$, Thioune Nene ${ }^{2}$, \\ David Carmen ${ }^{2,4}$, De La Chapelle Marc ${ }^{2}$, Compere Chantal ${ }^{1}$
}

1 IFREMER, Lab Detect Capteurs \& Mesures, CS10070, F-29280 Plouzane, France.

2 Univ Paris 13, UFR SMBH, Lab CSPBAT FRE 3043, 74 Rue Marcel Cachin, F-93017 Bobigny,

France.

${ }^{3}$ Univ Francois Rabelais Tours, UFR Sci Pharmaceut, EA Nanomedicaments \& Nanosondes 6295, 31

Ave Monge, F-37200 Tours, France.

${ }^{4}$ Horiba Sci, 231 Rue Lille, F-59650 Villeneuve Dascq, France.

*Corresponding author : Emmanuel Rinnert, email address : emmanuel.rinnert@ifremer.fr

\begin{abstract}
:
We demonstrated the use of a new organometallic nanoprobe for competitive surface-enhanced Raman scattering (SERS) immunoassay devoted to the detection of polycyclic aromatic hydrocarbons (PAH) such as benzo[a]pyrene (BaP) in seawater. The nanoprobes are gold nanoparticles (GNPs) labeled by a Raman reporter, the 5,5'-dithiobis(succinimidyl-2-nitrobenzoate) (DSNB) and functionalized with monoclonal antibodies anti-BaP. The antibodies are bound with a high specificity to the analyte while the GNPs enhanced the Raman scattering of the DSNB. This type of immunoassay involved the grafting of BaP onto a sensing surface. Thus, NH2-terminated self-assembled monolayer is formed on the surface of gold substrate using cysteamine. Amines finally reacted with 6-formylbenzo[a]pyrene. So, this SERS detection involves four steps: (i) the nanoprobes are incubated with the sample; (ii) a drop of the mixture is then put onto the substrate; (iii) the surface is rinsed; and (iv) the surface is analyzed by Raman spectroscopy. To synthesize the nanoprobes, firstly, we prepared GNPs according to Frens' method. Then, GNPs were spontaneously labeled by the DSNB Raman reporter, thanks to a strong gold-sulfur interaction. Thereafter, BaP antibodies were cross-linked to the DSNB labeled GNPs by reaction of proteins primary amino groups with $\mathrm{N}$-hydroxyl succinimide (NHS). Before use in SERS detection, their activity was controlled by surface plasmon resonance technique. The present method allows us to detect $\mathrm{BaP}$ at trace concentration $(2 \mathrm{nmol} / \mathrm{L})$. The results demonstrate that the proposed method has a great potential for application in the monitoring of seawater.
\end{abstract}

Keywords: Biosensor, Surface-enhanced Raman scattering, Polycyclic aromatic hydrocarbons, Benzo[a]pyrene antibody, Organometallic nanoprobe 


\section{Introduction}

Since the discovery by Fleischmann et al. in 1974 of a particularly intense enhancement of the Raman signal of pyridine molecules adsorbed on the roughened surfaces of silver electrodes, the scientific community has shown an ever increasing interest in the surface-enhanced Raman scattering (SERS) effect (Fleischmann et al. 1974). Very huge Raman enhancements, up to $10^{10}$-fold the normal Raman signal of molecules in bulk, have been observed when these molecules are adsorbed on active metallic surfaces (Fleischmann et al. 1976; Albrecht and Creighton 1977; Jeanmaire and Van Duyne 1977; Kneipp et al. 1997; Nie and Emory 1997; Guillot and Lamy de la Chapelle 2012). Consequently, SERS can be considered as an alternative to the inherent low cross-section of Raman scattering. In the investigation of chemical pollutions such as Polycyclic Aromatic Hydrocarbons at trace concentration (nmol/L), SERS effect reveals an immense potential regarding the environment as shown by the recent review made by Li et al. (2014).

The aim of the study is to give an innovative method to detect a PAH, the benzo[a]pyrene at low concentration in aqueous media. Such target molecule is of interest not only for the exploitation and exploration of oceanic and energetic resources but also for the monitoring of the quality of the environment. Nowadays, there is a lack of relevant technique that allows qualitative, quantitative, fast and in situ analysis of PAHs in aqueous media. The low solubility of PAHs in the aqueous media requires the development of not only sensitive but also selective sensors taking into account the variety of the encountered molecules in the media. Hydrophobic SERS-active substrates could offer the expected solution (Bao et al. 2012, Murphy et al. 1999; Péron et al. 2009a; Péron et al. 2009b; Péron et al. 2010; Pfannkuche et al. 2012; Schmidt et al. 2004). But, in order to ensure selectivity and sensitivity to the sensor, immunoassay is a powerful technique based on the specific interaction between the antigen and the complementary antibody. Coupled to SERS, immunoassay is already applied for medical diagnosis, biochemical analyses and environmental monitoring for macromolecules such as proteins and peptides (Xu et al. 2005). Through this work we demonstrate the feasibility of immuno-SERS detection system with small molecules such as PAHs.

Although silver presents a higher Raman enhancement factor compared to gold (10-100-fold greater), $\mathrm{Au}$ was chosen in this study to allow measurements in seawater on account of $\mathrm{AgCl}$ formation on silver surfaces (Murphy et al. 1997). The method of detection by competitiveness on a surface grafted with molecules of $\mathrm{BaP}$ is chosen. An organometallic complex assembling gold nanoparticles and antibodies is used. This complex is chemically designed using 5,5'-dithiobis(succinimidyl-2nitrobenzoate) (DSNB) as a bifunctional coupling agent. DSNB plays also a role of Raman reporter due to its particularly intense Raman signal (Grubisha et al. 2003). Grafted antigen on the sensor competes with free antigen in the sample for nanoprobe antibody binding sites. The more antigens 
there are in the sample the less organometallic nanoprobes are retained on the sensor surface resulting in a weaker signal. This article demonstrates the interest of developing sensitive sensors for seawater analyses with the aim of detecting PAHs. As far as we know and as it is confirmed in a review on recent progress in surface enhanced Raman spectroscopy for the detection of environmental pollutants, this paper relates for the first time benzo[a]pyrene detection coupling immunoassay and SERS (Li et al. 2014).

Thus, we present in this preliminary study, in addition to the methodology employed, techniques and test results of the various elements designed for the development of immuno-SERS system for the detection of PAHs. One example of an analysis is presented at the end of this work to show the feasibility of the method. Indeed, at this step, the detection is not optimized and the reproducibility study is not shown here.

\section{Materials and methods}

\subsection{Chemicals}

Benzo[a]pyrene (96\%), cysteamine (98\%), 2-mercaptoethanol (99\%), N-methylformanilide (99\%), Phosphorus oxychloride (99\%), sodium carbonate (99\%), sodium cyanoborohydride (95\%), methanol (99.8\%), 5,5'-dithiobis(2-nitrobenzoic acid) (DNBA) (99\%), 1,3-dicyclohexylcarbodiimide (99\%), albumine from bovine serum (BSA) (98\%) and $\mathrm{N}$-hydroxysuccinimide (NHS) (98\%) were obtained from Sigma Aldrich. N,N-dimethylformamide (99.8\%), sulfuric acid (95-98\%), tetrahydrofuran (p.a.) and boric acid (p.a.), were from Merck. Dimethyl ether (99.9\%) and sodium hydroxide solution at 10N (9.8-10-2 N) were from Fluka. Hydrogen peroxide solution (30\%wt in $\mathrm{H}_{2} \mathrm{O}$ ), dimethyl sulfoxyde (99.99\%), PBS were from Fisher. Acetonitrile (HPLC grade) and sodium tricitrate (reagent grade) were purchased from Prolabo. Antibody anti-benzo[a]pyrene (BaP13) was purchased from Exbio Antibodies. Gold III chloride trihydrate $\left(\mathrm{HAuCl}_{4} \cdot 3 \mathrm{H}_{2} \mathrm{O}\right)(>49 \%$ Au basis) was obtained from Acros organics. Water used in all experiments was purified using a Milli-Q ultrapure water purification system (Merck Millipore) with resistivity of $18 \mathrm{M} \Omega / \mathrm{cm}$.

\subsection{Instruments}

A home-made Surface Plasmon Resonance system was used. It is based on wavelength modulation in a configuration similar to the one designed by Cahill et al. (1997). The system was developed for laboratory and underwater experiments (Colas et al. 2010).

A NanoDrop 2000 Spectrophotometer (Thermo Scientific) was used to record UV-Vis Spectra of colloidal solutions in the spectral region between 350 and $800 \mathrm{~nm}$ (resolution $\leq 1.8 \mathrm{~nm}$ ). Analyzed solutions were diluted in distilled water $(10 \%, \mathrm{v} / \mathrm{v})$. Water spectrum was used as the baseline. The light source is a xenon flash lamp. 
A Horiba Jobin Yvon Labram HR800 spectrometer was used for recording the Raman and SERS spectra. Spectra were acquired with a $785 \mathrm{~nm}$ laser diode using a 300 grooves per $\mathrm{mm}$ grating and a x100 long working distance immersion microscope objective from Olympus (NA=1). Using a $800 \mathrm{~mm}$ spectrograph, the resolution was $2 \mathrm{~cm}^{-1}$. The laser power was estimated at $0.1 \mathrm{~mW}$ focused on an area of $1 \mu \mathrm{m}^{2}$.

\subsection{Synthesis procedures of organometallic nanoprobe}

To quantify benzo[a]pyrene contamination on seawater, we chose the method of detection by competitiveness. An organometallic complex assembling gold nanoparticles and antibodies $\mathrm{BaP} 13$ is used as a nanoprobe (Scheme 1a). This assembly is performed chemically using a bi-functional coupling agent called DSNB (5,5'-dithiobis (succinimidyl-2-nitrobenzoate). This molecule also plays the role of the reporter due to its particularly intense Raman signal around $1330 \mathrm{~cm}^{-1}$ (Grubisha et al. 2003). This nanoprobe is bring together with the BaP antigen of water sample (Scheme $1 \mathrm{~b}$ ). The free antibody binding sites of the nanoprobes are then available to react with grafted $\mathrm{BaP}$ on a gold coated quartz substrate (Scheme 1c). In this configuration, the grafted $\mathrm{BaP}$ competes with sample free antigen for nanoprobe antibody binding sites. The more antigens there are in the sample the less organometallic nanoprobes are retained in the surface sensing resulting in a weaker signal.

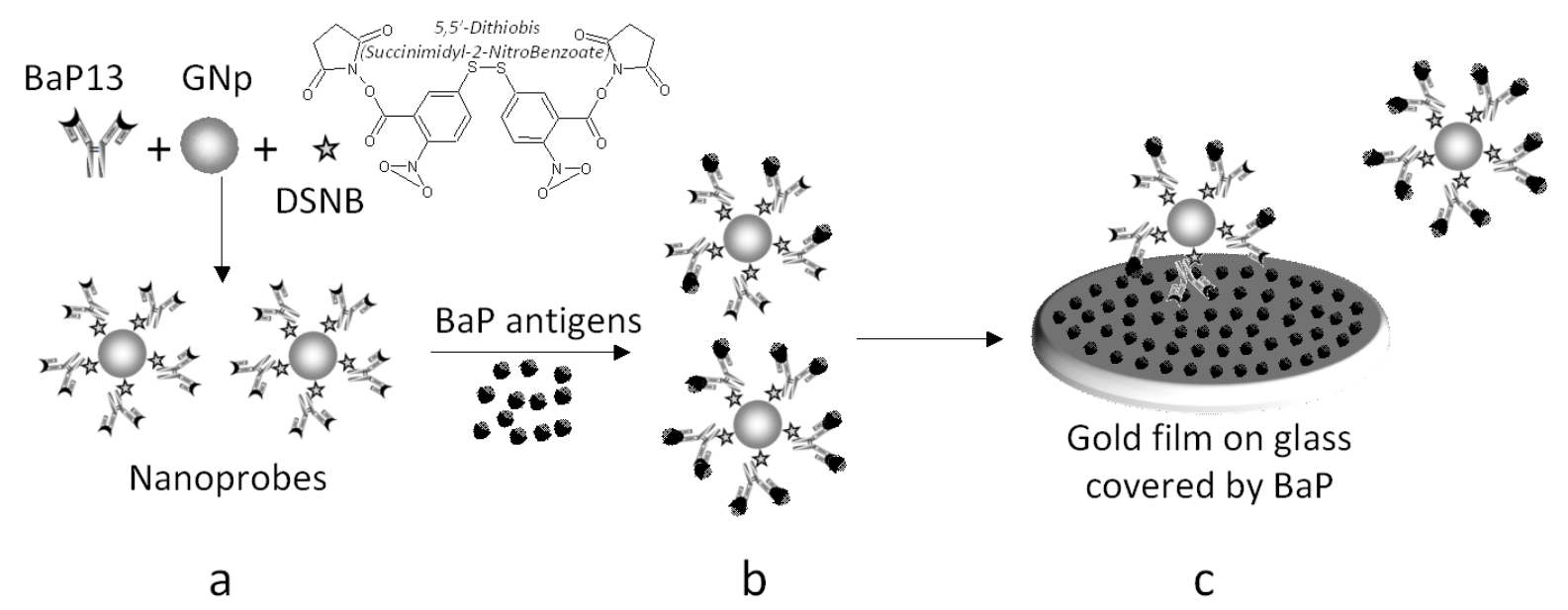

\subsubsection{Synthesis of 6-benzo[a]pyrenecarboxaldehyde}

Direct synthesis of 6-benzo[a]pyrenecarboxaldehyde from $\mathrm{BaP}$ was accomplished via VilsmeierHaack reaction using $\mathrm{N}$-methylformanilide and $\mathrm{POCl}_{3} . \mathrm{POCl}_{3}(1.2 \mathrm{~g})$ and $\mathrm{N}$-methylformanilide $(1.2 \mathrm{~g})$ were added to a solution of benzo[a]pyrene $(1 \mathrm{~g})$ in DMF $(10 \mathrm{~mL})$ in a $90 \mathrm{~mL}$ pyrex bottle. The screw cap was then sealed, and the reaction bottle was heated at $90^{\circ} \mathrm{C}$ overnight. The reaction mixture was cooled and washed with $40 \mathrm{~mL}$ of diethyl ether. The red slurry was recovered and mixed with $\mathrm{Na}_{2} \mathrm{CO}_{3}$ ( $2.5 \mathrm{~g} / 50 \mathrm{~mL}$ in water) in a $150 \mathrm{~mL}$ beaker. The green precipitate that formed was filtered, washed copiously with water, and then left dry at room temperature (Dai et al. 2007).

\subsubsection{Functionalization of the sensor surface with a SAM presenting BaP head groups}


Attachment of $\mathrm{BaP}$ to a gold surface of the sensor begins with a self-assembled mixed monolayer formation of cysteamine on this surface. This step was assured by means of the gold-alkanethiolate self-assembly process using cysteamine and 2-mercaptoethanol as described as fellows. First, substrate was cleaned in a piranha solution $\left(\mathrm{H}_{2} \mathrm{SO}_{4} / \mathrm{H}_{2} \mathrm{O}_{2} 30 \%-3: 1 \mathrm{v} / \mathrm{v}\right)$ at $80^{\circ} \mathrm{C}$ for 4 hours to remove organic contaminants. Next, the gold surface of the sensor chip was immersed in methanol solution of $5 \mathrm{mM}$ cysteamine and $5 \mathrm{mM} 2$-mercaptoethanol for 4 hours. The sensor chip is then washed with methanol to remove any adhered/loosely bound alkanethiol molecules from the sensor chip. Afterwards, the substrate was rinsed in DMSO and treated in DMSO solution of 6-benzo[a]pyrenecarboxaldehyde $(0.68 \mathrm{mg} / \mathrm{mL})$ for two hours. This forms a Schiff base which was then reduced by reaction of $14 \mu \mathrm{L}$ per $\mathrm{mL}$ of DMSO of a sodium cyanoborohydride solution $5 \mathrm{M}$ in $\mathrm{NaOH} 1 \mathrm{M}$ for one hour. Finally, grafted gold substrate was rinsed in distilled water and dried under a stream of nitrogen gas.

\subsubsection{Synthesis of 5,5'-Dithiobis(succinimidyl-2-nitrobenzoate) (DSNB)}

DSNB is one of derivatives of dithiobis(benzoic acid), which could easily be converted to the corresponding succinimide ester with NHS. The formula of DSNB is shown on Scheme 1. DSNB is a particularly attractive example because of the strong scattering cross section of its symmetric $\mathrm{NO}_{2}$ stretch. To obtain this compound, we activated carboxylic functions of DNBA using a reagent mixture carbodiimide / succinimide. So, $50 \mathrm{~mL}$ of dried tetrahydrofuran were added to $0.50 \mathrm{~g}$ of DNBA, $0.52 \mathrm{~g}$ of DCCD, and $0.29 \mathrm{~g}$ of NHS in a $100 \mathrm{~mL}$ round-bottom flask equipped with a drying tube. The mixture was magnetically stirred at $25{ }^{\circ} \mathrm{C}$ for $12 \mathrm{~h}$, filtered, and then rotaevaporated to remove solvent. The crude product was dissolved in $100 \mathrm{~mL}$ of acetonitrile and filtered yielding a yellow solution. We denote by $\mathrm{C}_{0}$, the DSNB concentration of this solution. This concentration is equal to 2.5 $\mathrm{mM}$ if the yield of the DNBA activation was equal to $100 \%$.

\subsubsection{Preparation of Raman Reporter-Labeled Immunogold colloids}

Suspensions of gold nanoparticles were prepared according to the method described by Frens (1973). Our preparation technique has been used in different studies and consists in the reduction of $\mathrm{HAuCl}_{4}$ by sodium tricitrate (Péron et al. 2009b; Péron et al. 2010). Here, $10 \mathrm{~mL}$ of sodium tricitrate at $8 \times 10^{-3}$ $\mathrm{M}$ was slowly added to $10 \mathrm{~mL}$ of boiling tetrachloroauric acid at $2 \times 10^{-3} \mathrm{M}$ while stirring. The reactor was placed on a hot plate and magnetically stirred. The mixture of both solutions was refluxed for $1 \mathrm{~h}$. After $1 \mathrm{~h}$ boiling, the mixture was cooled at room temperature while stirring.

The gold colloidal nanoparticles are coated by the DSNB derivative thanks to its thiolate. The primary amines of an antibody can be then linked by the formation of an amide linkage. The particle workup consisted in two steps. In step one, $100 \mu \mathrm{L}$ of a $2.5 \mathrm{mM}$ DSNB solution in acetonitrile was added to 1 $\mathrm{mL}$ of the unconjugated colloidal gold suspension and the mixture reacted for 3-5 h. The reporterlabeled colloids were then separated from solution by centrifugation at $10000 \mathrm{~g}$ for $7 \mathrm{~min}$. The clear 
supernatant was discarded, and the loose red sediment was resuspended in $1 \mathrm{~mL}$ of borate buffer (2 $\mathrm{mM}, \mathrm{pH}$ 9). In step two, BaP13 was coupled to the gold particles via the succinimidyl terminal of the DSNB-derived coating. As such, $35 \mu \mathrm{g}$ of detection antibody ( $35 \mu \mathrm{L}$ of $1 \mathrm{mg} / \mathrm{mL}$ BaP13 solution) was added to the $1 \mathrm{~mL}$ suspension of the reporter-labeled colloid. The mixture was then incubated at room temperature for $1 \mathrm{~h}$. After centrifugation at $10000 \mathrm{~g}$ for $7 \mathrm{~min}$ and removal of the supernatant, the red sediment was resuspended in $1 \mathrm{~mL}$ of PBS (pH 7.0, 1\% BSA) (Grubisha et al. 2003).

\subsection{SERS immunoassay procedure}

Raman analysis is performed using the following steps: firstly, we diluted the probe suspension obtained according to the protocol described above with 6 volumes of PBS. After that, we mixed one volume of the suspension thus obtained with one volume of seawater containing benzo[a]pyrene at a predefined concentration and incubate for 30 minutes. Thus, the molecules of $\mathrm{BaP}$ present in the sample to analyze will block the antigenic sites of antibodies in the organometallic probes. Then we placed a microdrop $(60 \mu \mathrm{L})$ of this mixture on the surface of a substrate previously covered with benzo[a]pyrene. We rinsed the substrate with water 30 minutes after the deposit and finally we analyzed with the Raman spectrometer the surface substrate using 100x water immersion objective.

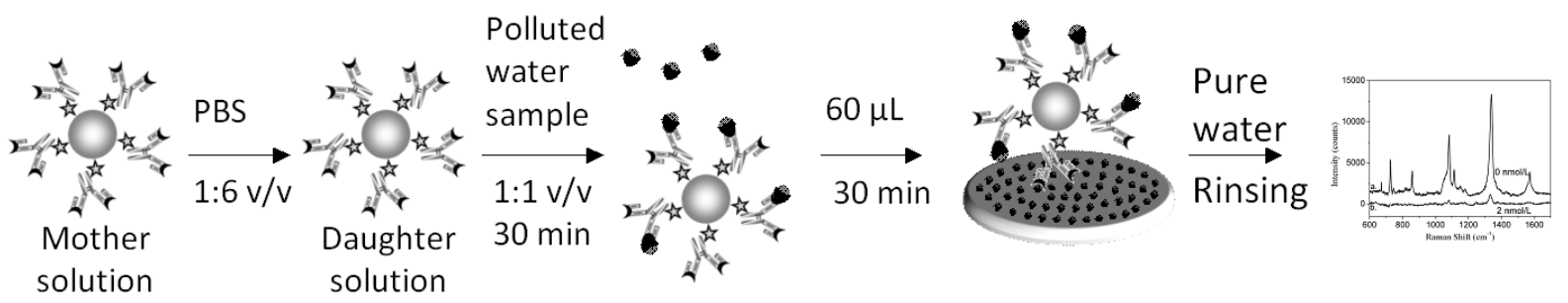

\section{Results and discussion}

\subsection{Surface grafting control of BaP coated substrate}

In order to control the surface grafting of benzo[a]pyrene, we have measured the binding ability of anti-BaP antibodies to the $\mathrm{BaP}$ functionalized substrate. So, for this, we used surface plasmon resonance (SPR) spectroscopy. Thus, we have studied two types of surface, functionalized and nonfunctionalized or uncoated surfaces. The analysis began with the injection of phosphate buffer at $\mathrm{pH} 7$. The BaP13 antibodies at $50 \mathrm{ppm}$ in PBS was then injected. With the two surfaces, we observed an increase in the SPR signal just after injection of antibodies in the measuring cell (Figure 1). However, we have noted that the increase obtained with the functionalized surface is considerably higher. Indeed, after PBS rinsing, the SPR profile obtained with the functionalized surface is characteristic for specific ligand-receptor interaction. Moreover, the measured offset is much higher with the functionalized surface $(\Delta \lambda \square=7.8 \mathrm{~nm})$ compared with the uncoated surface $(\Delta \lambda=0.4 \mathrm{~nm})$ (Figure 1). 
Thus, we can maintain the presence of $\mathrm{BaP}$ molecules on the surface of functionalized substrate and therefore the effectiveness of our protocol for benzo[a]pyrene grafting on the gold.

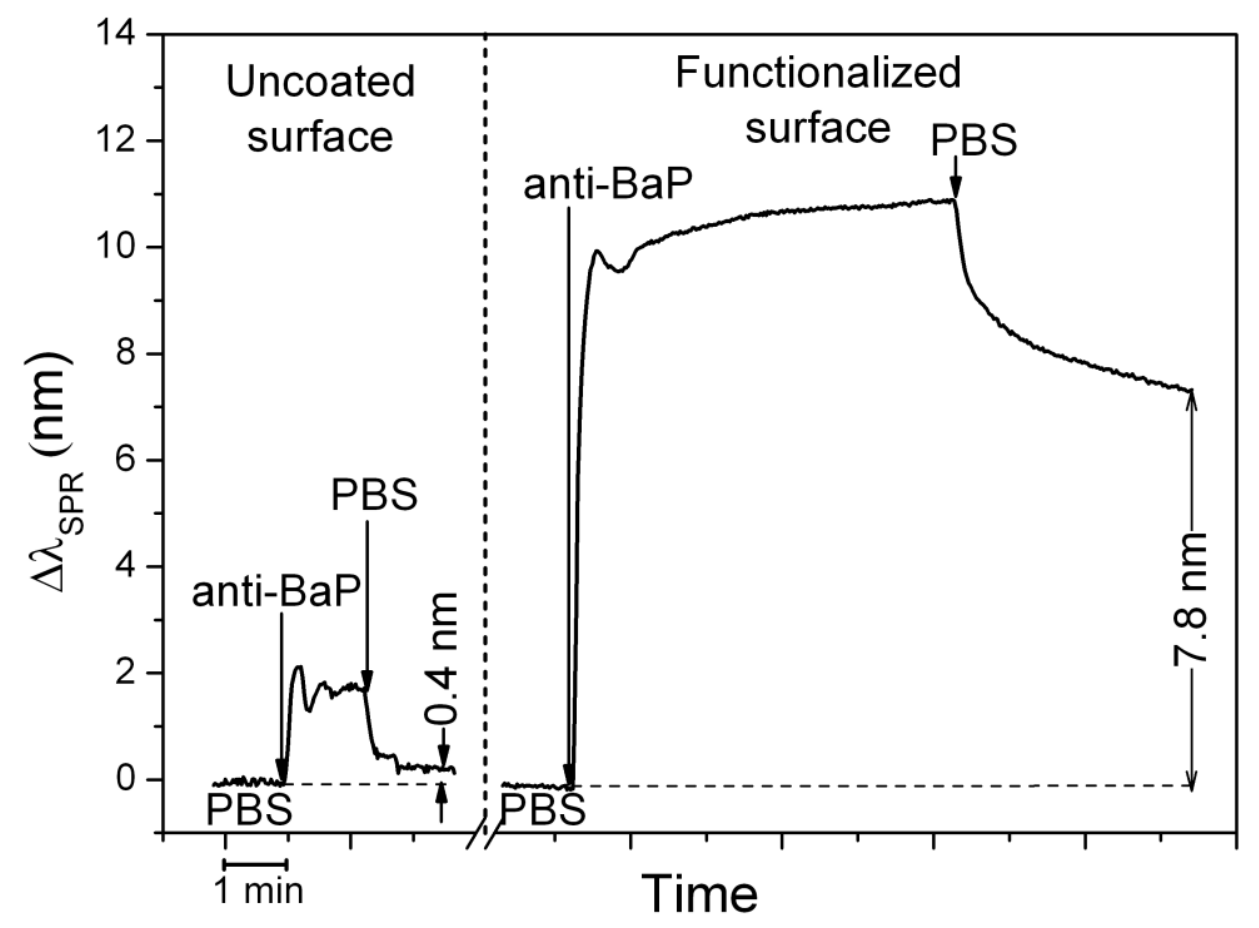

\subsection{Determination of size and concentration of gold nanoparticles from UV-Vis Spectra}

\subsubsection{Size}

We have determined the size of nanoparticles by measuring the wavelength of plasmon resonance. Indeed, a relationship was demonstrated between the optical absorption spectrum (in particular, the plasmon resonance) and the size of the spherical gold nanoparticles (Link and El-Sayed 2000). Thus, we have analyzed with the spectrophotometer the solution of gold nanoparticles obtained using the protocol described above. The resonance wavelength of this solution is equal to $525 \mathrm{~nm}$ (Figure 2). The displaying of spectra of nanoparticles solutions with sizes ranging from 9 to $99 \mathrm{~nm}$ allows us to determine that the diameter of our nanoparticles is between 22 and $48 \mathrm{~nm}$. 


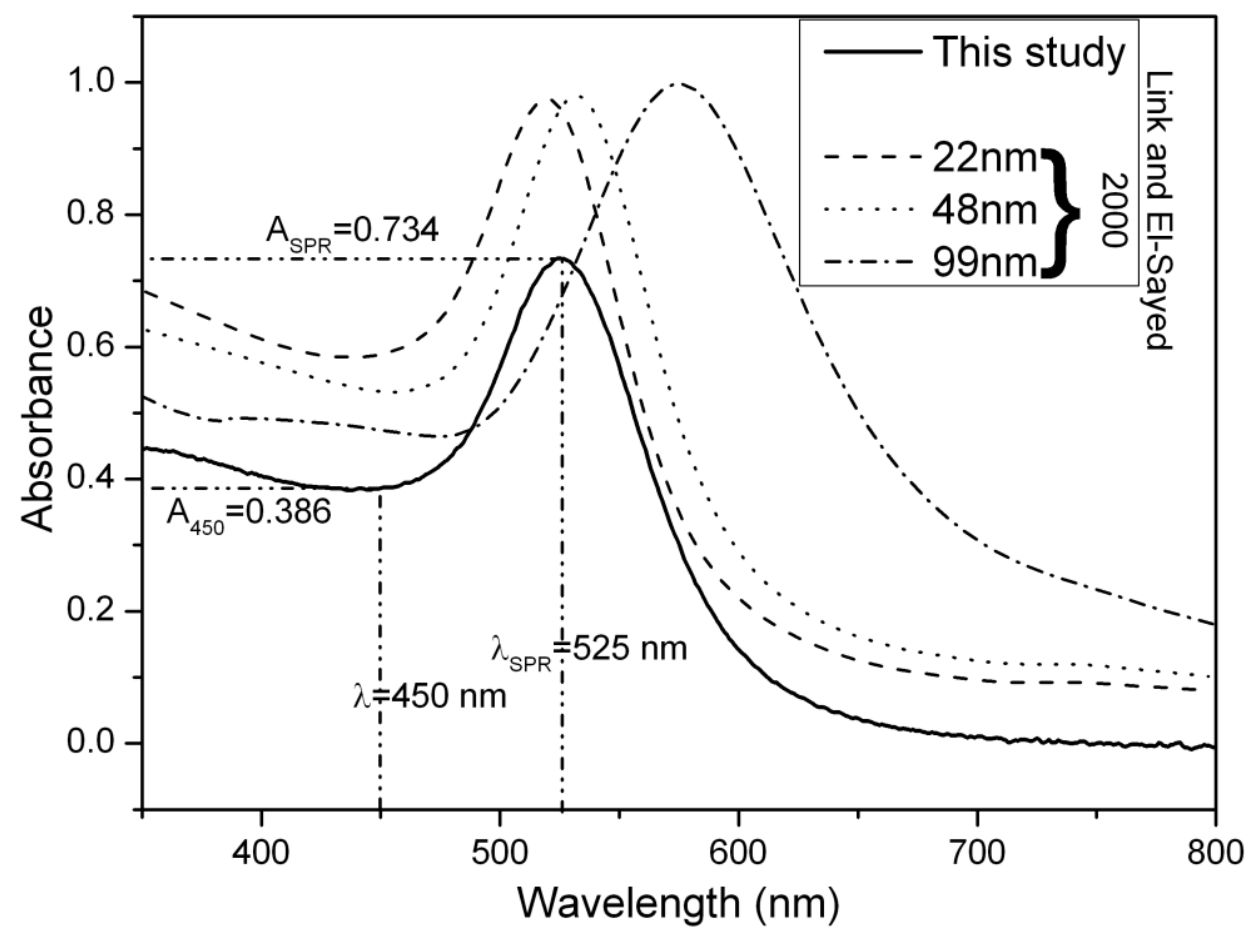

The dependence of the optical properties of spherical gold nanoparticles on particle size were analyzed by Haiss et al.(Haiss et al. 2007). They have succeeded in establishing a mathematical relationship between the diameter $(d)$ of gold nanoparticles and the absorption values of the corresponding solution at defined wavelengths.

$$
d=\exp \left(B_{1} * A_{S P R} / A_{450}-B_{2}\right)(\text { Haiss et al. 2007) }
$$

With $A_{S P R}$ the absorbance at the surface plasmon resonance peak and $A_{450}$ the absorbance at $450 \mathrm{~nm}$. $B_{1}$ and $B_{2}$ values are 3 and 2.2 respectively (Haiss et al. 2007).

With the absorbance of 0.734 and 0.386 at respective wavelengths of $525 \mathrm{~nm}$ and $450 \mathrm{~nm}$ (thick line in Figure 2), the average diameter of our nanoparticles calculated using this relationship is equal to 33 $\mathrm{nm}$.

\subsubsection{Number density of the GNPS}

To estimate the concentration of nanoparticles of our nanoparticles solution, we have established a relationship between the number density of the GNPs and their average size. Indeed, if we consider that the whole quantity of gold used for the preparation of nanoparticles has been consumed Pluchery and Carrière (2011), we can express the number density $n$ of nanoparticles in solution using the following equation: 


$$
n=V_{T} / V_{P}=3 * m /\left(4 * \rho^{*} \pi * r^{3} * V_{S}\right)
$$

with $V_{T}$ the volume of all gold nanoparticles, $V_{P}$ the average volume of one nanoparticle, $m$ the total mass of used gold, $\rho$ the density of gold $\left(19.28 \mathrm{~g} . \mathrm{cm}^{-3}\right)$, and $V_{S}$ the solution volume.

Thus, with an average diameter of about $33 \mathrm{~nm}$, the nanoparticles that we have prepared are dispersed with a number density of about $5.4 \times 10^{11}$ particles $/ \mathrm{mL}$.

\subsection{SERS assay of nanoprobes}

Prepared DSNB and the nanoprobe made of DNSB and antibodies were analyzed by Raman spectroscopy. The spectra of the range 600 and $1800 \mathrm{~cm}^{-1}$ are presented in figure 3. The free DSNB spectrum reported in figure 3.a. is obtained by subtracting the spectrum of the pure solvent from that of the acetonitrile solution. This spectrum is dominated by the symmetric nitro stretch at $1346 \mathrm{~cm}^{-1}$. The band at $1572 \mathrm{~cm}^{-1}$ is assigned to an aromatic ring mode, and the band at $1081 \mathrm{~cm}^{-1}$ is a succinimidyl N-C-O stretch overlapping with aromatic ring modes (Grubisha et al. 2003). The same bands in the solution spectrum are also observable in the spectrum of the DSNB nanoprobes (Figure 3.b.), although $\mathrm{NO}_{2}$ signal has undergone a shift which is probably assigned to the solvant effect due to the $\mathrm{C} \equiv \mathrm{N}$ group of acetoniltrile. We were especially interested in the higher intensity bands to estimate the enhancement factor obtained with the Raman probes that we have prepared. Taking into account the areas of the symmetric nitro stretch bands and the concentration ratio of DSNB for each sample, an enhancement factor equal to about 36000 is calculated. In order to make possible this calculation, the experimental conditions were the same for each sample (time of exposition, laser power, ...).This estimation assumes that the recovery rate of gold nanoparticles by reporter molecules is equal to one molecule per $\mathrm{nm}^{2}$. 


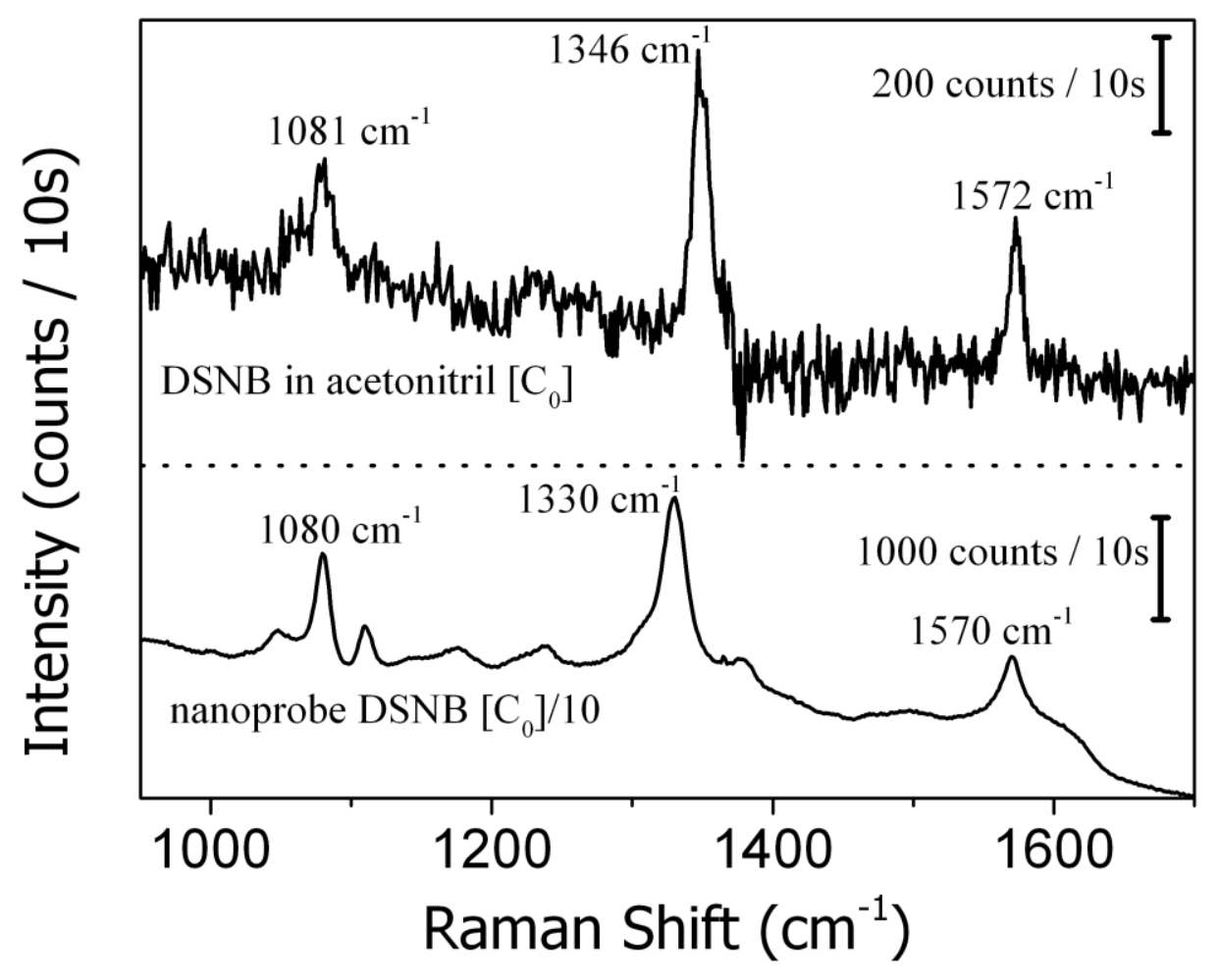

\subsection{Nanoprobe activity by Surface Plasmon Resonance sensor}

We have studied the nanoprobe activity by Surface Plasmon Resonance (SPR) spectroscopy using a gold surface covered with $\mathrm{BaP}$. We observed that the coupling of BaP13 antibodies to gold nanoparticles did not affect their affinity for the molecules of benzo[a]pyrene.

\subsection{BaP detection}

Figure 4 presents the results obtained with concentrations of $\mathrm{BaP} 0$ and $2 \mathrm{nmol} / \mathrm{L}$. When there is no $\mathrm{BaP}$ in the sample, the grafted antibodies onto the nanoparticles recognize and adsorb the $\mathrm{BaP}$ molecules immobilized at the surface of the sensor. Hence, the signal of reporter on spectrum (a.) in Figure 4 is important. On this spectrum, we observe the Raman bands related to $\mathrm{C}-\mathrm{H}$ (from 700 to $1000 \mathrm{~cm}^{-1}$ ), C-N (from 1000 to $1200 \mathrm{~cm}^{-1}$ ), $\mathrm{N}-\mathrm{O}$ (from 1300 to $1400 \mathrm{~cm}^{-1}$ ) and $\mathrm{C}=\mathrm{C}$ (from 1500 to $1600 \mathrm{~cm}^{-1}$ ) bonds of DSNB. These bands are nearly extinct in the spectrum (b.) in Figure 4 obtained in the presence of $\mathrm{BaP}$ at the concentration of $2 \mathrm{nmol} / \mathrm{L}$. This is perfectly in accordance with the principle of the sensing method. Indeed the BaP molecules in the analyzed sample inhibit Raman probes to adsorb to the sensor surface by blocking the antigenic sites free of antibodies at the surface of these probes. Hence a significant attenuation of the SERS effect was observed. However, it is important to note that this result suffers of a lack of reproducibility. Indeed, a more advanced work on the effectiveness of the analysis is underway to solve this problem. 


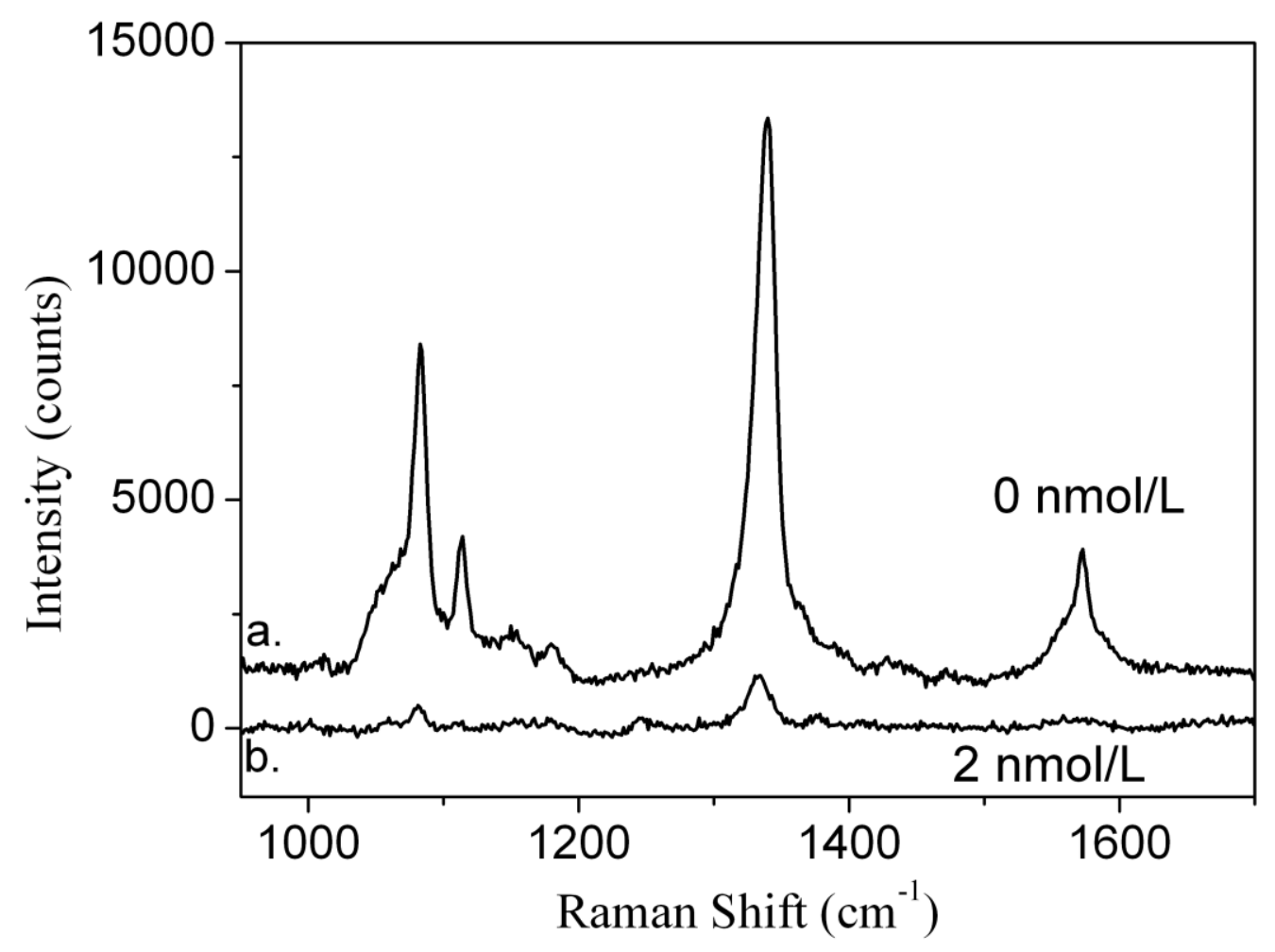

\section{Conclusions}

The present work has demonstrated an efficient method to prepare surface sensor and SERS nanoprobes for specific detection of benzo[a]pyrene in seawater. The 6benzo[a]pyrenecarboxaldehyde was used to modify the sensor surface for competitive assay. The SERS enhancement is observed through organometallic nanoprobes that were obtained by coupling of GNPs, DSNB and BaP13. Quantitative determination of $\mathrm{BaP}$ at nanomolar level was achieved. However, works on improving reproducibility are necessary in order to confirm the effectiveness of this detection method. This method has already the advantages of an approach that does not require extraction steps or large sample volumes. This should have great applications in rapid quantification of PAHs in complex. The sensitivity of this analytical method will open new ways toward a generalized use of our technique, not only in the laboratory but also in field assays with a portable Raman spectrometer.

\section{Acknowledgments}

This project was financing by ANR, the French Research Agency thanks to the Ifremer-Edrome Carnot Institute Grants and thanks to the ECOTECH 2011 program, through the ANR-11-ECOT-010REMANTAS project. 


\section{References}

Albrecht MG and Creighton JA (1977) Anomalously intense Raman spectra of pyridine at a silver electrode. J. Am. Chem. Soc. 99:5215-5217. doi: 10.1021/ja00457a071

Bao L, Sheng P, Li J, Wu S, Cai O, Yao S (2012) Surface enhanced Raman spectroscopic detection of polycyclic aromatic hydrocarbons (PAHs) using a gold nanoparticles-modified alginate gel network. Analyst 137:40104015. doi: $10.1039 / \mathrm{C} 2 \mathrm{AN} 35589 \mathrm{~B}$

Cahill CP, Johnson KS, Yee SS (1997) A surface plasmon resonance sensor probe based on retro-reflection. Sensors and Actuators B: Chemical 45:161-166. doi: 10.1016/S0925-4005(97)00290-6

Colas F, Crassous MP, Litaker W, Laurent S, Rinnert E, Compère C, Gentien P (2010) New Approach For Direct Detection Of Domoic Acid. Proceedings of the 14th international conference on harmful algae, Hersonissos-Crete, Greece, 1-5 November 2010.

Dai Q, Xu D, Lim K, Harvey RG (2007) Efficient Syntheses of C8-Aryl Adducts of Adenine and Guanine Formed by Reaction of Radical Cation Metabolites of Carcinogenic Polycyclic Aromatic Hydrocarbons with DNA. J. Org. Chem. 72(13):4856-4863. doi: 10.1021/jo070518m

Fleischmann M, Hendra PJ, McQuillan AJ (1974) Raman spectra of pyridine adsorbed at a silver electrode. Chem. Phys. Lett. 26:163-166. doi: 10.1016/0009-2614(74)85388-1

Fleischmann M, Hendra PJ, McQuillan AJ, Paul RL, Reide ES (1976) Raman spectroscopy at electrodeelectrolyte interfaces. J. Raman Spectrosc. 4:269-274. doi: 10.1002/jrs.1250040308

Frens G. (1973) An experiment concerning the dispersion forces between very small metal spheres. Physics Letters A 44(3):208-210. doi: 10.1016/0375-9601(73)90885-2

Grubisha DS, Lipert RJ, Park HY, Driskell J, Porter MD (2003) Femtomolar Detection of Prostate-Specific Antigen: An Immunoassay Based on Surface-Enhanced Raman Scattering and Immunogold Labels. Anal. Chem. 75:5936-5943. doi: 10.1021/ac034356f

Guillot N and Lamy de la Chapelle M (2012) The electromagnetic effect in surface enhanced Raman scattering: Enhancement optimization using precisely controlled nanostructures. Journal of Quantitative Spectroscopy and Radiative Transfer 113(18):2321-2333. doi: 10.1016/j.jqsrt.2012.04.025

Haiss W, Thanh NTK, Aveyard J, Fernig DG (2007) Determination of Size and Concentration of Gold Nanoparticles from UV-Vis Spectra. Anal. Chem. 79(11):4215-4221. doi: 10.1021/ac0702084

Jeanmaire DJ and Van Duyne RP (1977) Surface raman spectroelectrochemistry. J. Electroanal. Chem. 84:1-20. doi: 10.1016/S0022-0728(77)80224-6

Kneipp K, Wang Y, Kneipp H, Perelman LT, Itzkan I, Dasari RR, Feld MS (1997) Single Molecule Detection Using Surface-Enhanced Raman Scattering (SERS). Phys. Rev. Lett. 78:1667-1670. doi: 10.1103/PhysRevLett.78.1667

Li DW, Zhai WL, Li YT, Long YT (2014) Recent progress in surface enhanced Raman spectroscopy for the detection of environmental pollutants. Microchimica Acta 181(1-2):23-43. doi: 10.1007/s00604-013-1115-3

Link S and El-Sayed MA (2000) Shape and size dependence of radiative, non-radiative and photothermal properties of gold nanocrystals. International Reviews in Physical Chemistry 19(3):409-453. doi: $10.1080 / 01442350050034180$

Murphy T, Schmidt H, Kronfeldt HD (1997) Detection of chemicals in seawater using surface-enhanced Raman scattering (SERS). SPIE 3107:281-287. doi: 10.1117/12.274727 
Murphy T, Schmidt H, Kronfeldt HD (1999) Use of sol-gel techniques in the development of surface-enhanced Raman scattering (SERS) substrates suitable for in situ detection of chemicals in sea-water. Appl. Phys. B: Lasers Opt. 69(2):147-150. doi:10.1007/s003400050787

Nie S and Emory SR (1997) Probing Single Molecules and Single Nanoparticles by Surface-Enhanced Raman Scattering. Science 275:1102-1106. doi:10.1126/science.275.5303.1102

Péron O, Rinnert E, Lehaitre M, Colas F, Compère C (2009a) Towards in situ detection of PAH trace in seawater using SERS-active sensors. Proceeding of SPIE Defense, Security \& Sensing 7312:73120D1-10. doi: $10.1117 / 12.818641$

Péron O, Rinnert E, Lehaitre M, Crassous P, Compère C (2009b) Detection of polycyclic aromatic hydrocarbon (PAH) compounds in artificial sea-water using surface-enhanced Raman scattering (SERS). Talanta 79:199-204. doi: 10.1016/j.talanta.2009.03.043

Péron O, Rinnert E, Lehaitre M, Colas F, Compère C (2010) First Steps of in Situ Surface-Enhanced Raman Scattering During Shipboard Experiments. Appl. Spectrosc. 64(10):1086-1093.

Pfannkuche J, Lubecki L, Schmidt H, Kowalewska G, Kronfeldt HD (2012) The use of surface-enhanced Raman scattering (SERS) for detection of PAHs in the Gulf of Gdańsk (Baltic Sea). Marine Pollution Bulletin 64(3):614-626. doi: 10.1016/j.marpolbul.2011.12.008

Pluchery O and Carriere M (2011) Nanoparticules d'or. Dossiers Techniques de l'Ingénieur NM900, TI-WEKA, Paris.

Schmidt H, Bich Ha N, Pfannkuche J, Amann H, Kronfeldt HD, Kowalewska G (2004) Detection of PAHs in seawater using surface-enhanced Raman scattering (SERS). Marine Pollution Bulletin 49(3):229-234. doi: 10.1016/j.marpolbul.2004.02.011

Xu SP, Ji XH, Xu WQ, Zhao B, Dou XM, Bai YB, Ozak Y (2005) Surface-enhanced Raman scattering studies on immunoassay. J. Biomed. Opt 10(3):031112-. doi: 10.1117/1.1915487 